\title{
Predictors of vasovagal reactions during preoperative autologous blood donation: A single-institution
}

analysis

Hisakazu Nishimori ${ }^{1,2}$, Nobuharu Fujii ${ }^{1}$, Keiko Fujii ${ }^{1}$, Tohru Ikeda $^{1}$, Naomi Asano ${ }^{1}$, Hiroaki Ogo ${ }^{1}$, Miwa Yamakawa $^{3}$, Naoe Takagi ${ }^{3}$, Fumio Otsuka ${ }^{1}$, and Kazuma Ikeda ${ }^{1,4}$

${ }^{1}$ Department of Transfusion Medicine, Okayama University Hospital, Okayama, Japan

${ }^{2}$ Department of Hematology and Oncology, Okayama University Hospital, Okayama, Japan

${ }^{3}$ Department of Nursing, Okayama University Hospital, Okayama, Japan

${ }^{4}$ Japanese Red Cross Okayama Blood Center, Okayama, Japan

\section{Corresponding author:}

Hisakazu Nishimori, M.D., Ph.D.

Department of Hematology and Oncology, Okayama University Hospital, 2-5-1 Shikata-cho, Kita-ku, Okayama, 700-8558 Japan.

Tel: 81-86-235-7227

Fax: 81-86-232-8226

E-mail: n-mori@md.okayama-u.ac.jp

Running head: VVRs during autologous blood donation

Type of manuscript: original article 


\section{Abstract}

Studies examining risk factors associated with vasovagal reactions (VVRs) during autologous blood donations, especially in younger subjects, have been limited. The aim of the present study was to define risk factors for VVRs during preoperative autologous blood donation in patients, including those younger than 18 years old. We retrospectively analyzed 4,192 autologous, preoperative blood donations between 2007 and 2015 at Okayama University Hospital. Eighty-seven (2.08\%) of the patients experienced VVRs. VVRs occurred approximately three times as often in patients $0-17$ years old $(16 / 320,5.0 \%)$ than in patients 18 years and older $(71 / 3,872$, 1.8\%). In particular, VVRs occurred more frequently in those 10-13 years old, and decreased with older age ( $P$ $=0.006$ ). In a univariate analysis, younger age, lower body mass index, lower systolic blood pressure, lower body weight, lower total blood volume, female gender, first-time collection, and higher heart rate were associated with a higher incidence of VVRs. In a multivariate analysis, lower systolic blood pressure $(P<$ 0.001), higher heart rate $(P=0.007)$, and first-time collection $(P=0.015)$, remained independent predictors of VVRs. These results emphasize the need for careful attention during blood collection.

Keywords: Autologous blood donation, vasovagal reactions 


\section{Introduciton}

Autologous blood donation is widely used for preoperative blood preparation to reduce exposure to allogeneic blood[1]. Autologous and allogeneic blood donors occasionally experience adverse reactions during or after blood withdrawal, such as weakness, pallor, nausea, sweating, and fainting [2, 3]. These symptoms are generally called "vasovagal reactions" (VVRs)[2, 4]. However, the pathophysiology of VVRs is not yet completely understood[5]. Although risk factors for VVRs have been analyzed mainly in healthy allogeneic donors or peripheral blood stem cell donors [2, 4, 6-8], reports describing patients undergoing autologous collection have been limited. In particular, there are few data concerning patients younger than 16-18 years old. In the present study, we analyzed the risk factors for VVRs during autologous donation in patients, including those younger than 18 years old, at a single institution.

\section{Materials and methods}

We retrospectively collected medical information from 4,192 patients (groups I + II) who underwent autologous preoperative blood collection between July 2007 and June 2010 (group I, n = 1,649) and between July 2010 and June 2015 (groups II, $\mathrm{n}=2543$ ) at Okayama University Hospital in Okayama City, Japan. The study protocol was approved by the Institutional Review Board of Okayama University Hospital and complied with the provisions of the Declaration of Helsinki.

Patients were screened for vital signs, height, body weight, and complete blood count. Enrolled patients underwent whole blood phlebotomy in a designated room: blood volumes did not exceed $400 \mathrm{~mL}$, or [body weight] $/ 50 \times 400 \mathrm{~mL}$ if the patient's body weight was less than $50 \mathrm{~kg}$. All collections were conducted by a physician and nurse from the Department of Transfusion Medicine at Okayama University Hospital. An 18-gauge needle was inserted into an antecubital vein or a large forearm vein of each patient, and autologous blood was collected using a negative pressure blood drawing device (Hemoquick; Terumo, Tokyo, Japan). After phlebotomy, the patients were administered an intravenous infusion of saline, or consumed at least $400 \mathrm{~mL}$ of fluid for volume replacement. Most patients were scheduled for collection two or three times with 7-day intervals. The last collection was performed 3 or more days before the operation. In our protocol, the following exclusion criteria were applied: (1) a hemoglobin (Hb) level of $<10 \mathrm{~g} / \mathrm{dL}$ (patients with $10-11 \mathrm{~g} / \mathrm{dL}$ were 
included only when their baseline Hb level was 10-11 g/dL), (2) systolic blood pressure $>200 \mathrm{mmHg}$, or $<90$ $\mathrm{mmHg}$, (3) diastolic blood pressure $>120 \mathrm{mmHg}$ or $<50 \mathrm{mmHg}$, (4) unstable angina pectoris, recent myocardial infarction (within the previous 6 months), severe aortic stenosis, or severe dysrhythmia, (5) ongoing bacterial infection, (6) unmanageable diabetes, and (7) a history of uncontrolled convulsions. Body mass index (BMI) for each patient was calculated based on weight and height. Similarly, total blood volume (TBV) was calculated from the height and weight using gender-specific formulas from Nadler and colleagues [9].

All VVRs were recorded by the staff of the Department of Transfusion Medicine on a specifically designed form. VVRs were divided into mild [pallor, weakness, dizziness, sweating, nausea accompanied by bradycardia (> 40 beats/min; bpm), or hypotension (> $90 \mathrm{mmHg}$ ) decreased by more than $20 \mathrm{mmHg}$ compared to pre-donation], moderate [unconsciousness, vomiting, bradycardia $(\leq 40 \mathrm{bpm})$, or hypotension $(\leq 90 \mathrm{mmHg})$ in addition to mild symptoms], and severe (convulsion, tetany, or incontinence in addition to moderate symptoms) [10].

\section{Statistics}

The basic characteristics of each group (non-VVR and VVR) were compared using the $\chi 2$ test or Student's $t$-test, as appropriate. First, we analyzed group I, and then the differences in relation to group II and the overall group (groups I + II). Multivariate analyses were performed using a logistic regression model to define the independent impact of each variable on VVR incidence. Statistical tests were two-sided, and the differences were deemed to be statistically significant if $p<0.05$. Linear regression analysis was used to determine the correlation between age and susceptibility to VVRs; $R>0.7$ was considered to as a strong correlation while a $p$ $<0.05$ was considered statistically significant. Data were analyzed using STATA software (ver. 12; StataCorp, College Station, TX, USA).

\section{Results}

During the study period, 4,192 autologous whole blood donations were made. Group I was comprised of 1,649 patients who donated between July 2007 and June 2010, and group II was comprised of 2,543 patients who donated between July 2010 and June 2015. Table 1 shows the patient background data. Most of the patients were females, and were being treated for orthopedic or gynecological diseases. The median patient age was 54 (range 
4-86) years. This study included 319 young patients ranging in age from 0 to 17 years. There were 44 VVRs $(2.67 \%)$ in group I, 43 VVRs (1.69\%) in group II, and 87 VVRs (2.08\%) in groups I + II. Place, time and description of blood donation for the patients did not differ between groups I and II. However, the doctors and staff differed between groups I and II. Doctors' skill and the accumulation of staff knowledge or experience might partly influence the occurrence of VVRs.

Severe, moderate, and mild VVRs were observed in six (6.9\%), two (2.3\%), and 79 (90.8\%) patients, respectively. Figure 1 shows the distributions of each VVR event grade by age. There was no increased susceptibility to severe VVRs with age. Figure 2 shows the VVR incidence rate by age. The VVR rate of 0-17-year-old patients was approximately three times as high as that of patients $18-89$ years old $(p<0.001)$. Among the younger populations, the VVR rate of 10-13-year-old patients was the highest $(5 / 92,5.43 \%)$, followed by $14-17$-year-olds $(11 / 203,5.41 \%)$, and $18-19$ year-olds $(3 / 72,4.17 \%)$. However, there were no VVRs in patients under 10 years old. In the adult population, VVR susceptibility was significantly negatively correlated with increasing age (20-29 years, 5.0\%; 30-39 years, 4.0\%; 40-49 years, 1.6\%; 50-59 years, 1.4\%; 60-69 years, $0.8 \%$; and 70-79 years, $0.5 \% ; R=0.88, p=0.006)$.

Table 2 lists the characteristics of VVR and non-VVR patients. Univariate analyses revealed that younger age, lower BMI, lower pre-collection systolic blood pressure, lower body weight, lower TBV, female gender, first-time collection, and higher heart rates were associated with a significantly higher incidence of VVRs in group I. In group II, younger age, and lower pre-collection systolic blood pressure were the significant factors associated with VVRs. In the overall group, all of these factors except female gender remained associated with a higher incidence of VVRs.

To determine which variables were independent predictors of VVRs, a logistic regression analysis was performed using seven predictors (Table 3). In group I, First-time collection (odds ratio $(\mathrm{OR})=2.76,95 \% \mathrm{CI}=$ $1.31-5.81, p=0.007)$, younger age $(<18$ years old: $\mathrm{OR}=2.77,95 \% \mathrm{CI}=1.16-6.61, p=0.021)$, higher heart rate $(>74$ bpm: $\mathrm{OR}=2.15,95 \% \mathrm{CI}=1.06-4.38, p=0.035)$, and lower pre-collection systolic blood pressure $(<120$ mmHg: $\mathrm{OR}=2.17,95 \% \mathrm{CI}=1.05-4.49, p=0.037$ ) were significant independent predictors of VVRs. In group 
II, lower systolic blood pressure $(\mathrm{OR}=4.23,95 \% \mathrm{CI}=2.04-8.76, p<0.001)$ was the only significant independent predictor of VVRs. In the overall group, lower systolic blood pressure $(\mathrm{OR}=2.45,95 \% \mathrm{CI}=$ $1.50-4.01, p<0.001)$, higher heart rate $(\mathrm{OR}=1.92,95 \% \mathrm{CI}=1.20-3.09, p=0.007)$, and first-time collection $(\mathrm{OR}=1.81,95 \% \mathrm{CI}=1.12-2.92, p=0.015)$ remained significant predictors of VVRs. The populations in the overall group were larger than in group I and group II; therefore, the rates of VVRs and the independent predictors of VVRs in this study might reflect the true situation in the overall group.

\section{Discussion}

An important aim of this study was to assess the risk for VVRs in younger patients in particular, because their life expectancy is long, and thus they may obtain greater benefit from autologous blood transfusions than allogeneic blood transfusions. In this study, we included younger patients (4-9 years old: $n=25,10-17$ years old: $n=295$ ) than previous studies[11-16]. Some reports did not assess donors under 17 years of age, because they primarily analyzed allogeneic donors[2, 4, 6, 7]. Previous studies demonstrated the safety of autologous blood donation for pediatric cardiac surgery $(n=80)[13]$ and homologous donation from high school students[17], and the risk of VVR was reported to be higher in younger autologous or homologous blood donors $[2,3,11,15]$. Our data revealed that the patients most susceptible to developing VVR were 10-13 years old, followed by those aged 14-17 years. Eder et al. reported that 16- and 17-year-olds were significantly more likely to experience a loss of consciousness and major systemic (syncopal-type) complications $(0.53 \%)$ than 18 and 19-year-old donors $(0.33 \%)$, or donors aged 20 years or older $(0.08 \%)$ during allogeneic donation [18]. Our data add evidence that patients younger than 14 years of age are yet more susceptible to develop VVRs. Moreover, susceptibility to VVRs decreased significantly according to older age. However, there was no case of VVR under the age of 10 years, although we could not discuss this statistically because there were only 25 patients.

One possible reason that younger patients are at higher risk of VVRs is the relationship between ventricular baroreceptor sensitivity and age[2, 19]. Healthy, young patients have lower ventricular baroreceptor sensitivity when subjected to physical or psychological stress[2]. Carotid-aortic baroreceptor sensitivity, which correlates inversely with ventricular baroreceptor sensitivity, decreases with age[20]. This downregulates ventricular 
baroreceptor sensitivity in younger patients, possibly resulting in their higher risk of VVRs[2].

VVRs occur after overstimulation of the parasympathetic versus sympathetic nervous system [5]. These nervous systems may be affected by the patient's emotions. In fact, VVRs are mediated by emotions [21]. Moreover, it was reported that psychological stress suppressed ventricular baroreceptor sensitivity[22]; thus, nervous patients may be more susceptible to VVRs[4]. It is expected that patients who donate autologous blood for the first time would be more anxious than those who give repeat blood donations, simply because the procedure of blood donation is unknown to them. Higher heart rate might also correlate with patient stress or anxiety and thereby be a risk factor for VVRs.

In this study, pre-collection systolic blood pressure $<120 \mathrm{mmHg}$ was shown to be the most significant risk factor of VVRs. Ogata et al. demonstrated that lower diastolic blood pressure was associated with a higher VVR rate, explaining why younger donors have lower blood pressure [23]. However, there is a report showing that a lower systolic blood pressure of $<100 \mathrm{mmHg}$ was associated with a significantly lower VVR rate [24]. This discrepancy could be explained by the method of multivariate analysis; i.e., adjusted or not adjusted for factors including age $[23,24]$. In our analyses, the OR and $p$-value were $2.17(95 \% \mathrm{CI}=1.05-4.49)$ and 0.037 , respectively, in group I. In group II, lower systolic blood pressure revealed the only significant independent predictor of VVRs $(\mathrm{OR}=4.23,95 \% \mathrm{CI}=2.04-8.76, p<0.001)$. The additional analysis that included more patients (the overall group) revealed that lower systolic blood pressure was the strongest independent predictor of VVR $(\mathrm{OR}=2.45(95 \% \mathrm{CI}=1.50-4.01), p<0.001))$. Our data add evidence that patients with a lower blood pressure are more susceptible to VVRs.

The high risk groups for VVRs are generally reported to be young, female, first-time donors [2, 18, 25-27]. In our analysis, female gender was a significant risk factor in the univariate analysis in group I; however, it was not significant in the multivariate analysis in both group I and the overall group. This might be explained by the fact that more female patients were included in this study (females: 2,992, males: 1,200) compared with previous reports, resulting in less power to determine the susceptibility in males. 
One limitation of this study was its retrospective nature. Also, the populations in each age group were not large; thus, the rates of VVRs in this study may not reflect the true situation. For example, that there were no VVRs in the group aged under 10 years may be because more attention was paid to these patients. However, these data may be valuable because the peak of the risk, in terms of age, cannot be seen in data on healthy allogeneic donors.

In summary, risk factors for VVRs include lower systolic blood pressure, higher heart rate, and first-time collection. In younger population, the patients most susceptible to developing VVR were 10-13 years old, followed by those aged 14-17 years. Even in the adult patient population, younger age was correlated with susceptibility to VVRs. These data should provide both reassurance and caution to blood collection staff, patients, and physicians with regard to the safety and risks of autologous blood collection. It may be possible to prevent VVRs by diminishing the anxiety of autologous patients, especially among younger populations.

\section{Acknowledgments}

We thank Noboru Asada, Hideaki Fujiwara, Yujin Kobayashi, Yusuke Meguri, Tatsunori Ishikawa, Takanori Yoshioka, Mariko Endo, and Takako Kumamoto for their skillful management.

\section{Compliance with ethical standards}

Conflict of interest The authors have no potential conflict of interest to report related to this article. 


\section{References}

1. Gandini G, Franchini M, de Gironcoli M, Giuffrida A, Bertuzzo D, Zanolla L, et al. Preoperative autologous blood donation by elderly patients undergoing orthopaedic surgery. Vox Sang. 2001; 80: 95-100.

2. Trouern-Trend JJ, Cable RG, Badon SJ, Newman BH, Popovsky MA. A case-controlled multicenter study of vasovagal reactions in blood donors: influence of sex, age, donation status, weight, blood pressure, and pulse. Transfusion. 1999; 39: 316-20.

3. Tomita T, Takayanagi M, Kiwada K, Mieda A, Takahashi C, Hata T. Vasovagal reactions in apheresis donors. Transfusion. 2002; 42: 1561-6.

4. Kasprisin DO, Glynn SH, Taylor F, Miller KA. Moderate and severe reactions in blood donors. Transfusion. 1992; 32: 23-6.

5. Aydin MA, Salukhe TV, Wilke I, Willems S. Management and therapy of vasovagal syncope: A review. World J Cardiol. 2010; 2: 308-15.

6. Tomasulo PA, Anderson AJ, Paluso MB, Gutschenritter MA, Aster RH. A study of criteria for blood donor deferral. Transfusion. 1980; 20: 511-8.

7. Graham DT. Prediction of fainting in blood donors. Circulation. 1961; 23: 901-6.

8. Nakane T, Nakamae H, Koh H, Nakamae M, Aimoto R, Terada Y, et al. Heart rate variability during and after peripheral blood stem cell leukapheresis in autologous transplant patients and allogeneic transplant donors. Int J Hematol. 2010; 91: 478-84.

9. Nadler SB, Hidalgo JH, Bloch T. Prediction of blood volume in normal human adults. Surgery. 1962; 51: $224-32$

10. Crocco I, Franchini M, Garozzo G, Gandini AR, Gandini G, Bonomo P, et al. Adverse reactions in blood and apheresis donors: experience from two Italian transfusion centres. Blood Transfus. 2009; 7: $35-8$.

11. McVay PA, Andrews A, Kaplan EB, Black DB, Stehling LC, Strauss RG, et al. Donation reactions among autologous donors. Transfusion. 1990; 30: 249-52.

12. Kasper SM, Weimbs G, Sabatowski R, Wassmer G. A randomized crossover trial of IV fluid replacement versus no fluid replacement in autologous blood donors with cardiovascular disease. Transfusion. 2002; 42: 226-31.

13. Masuda M, Kawachi Y, Inaba S, Matsuzaki K, Fukumura F, Morita S, et al. Preoperative autologous blood donations in pediatric cardiac surgery. Ann Thorac Surg. 1995; 60: 1694-7.

14. Popovsky MA, Whitaker B, Arnold NL. Severe outcomes of allogeneic and autologous blood donation: frequency and characterization. Transfusion. 1995; 35: 734-7.

15. AuBuchon JP, Popovsky MA. The safety of preoperative autologous blood donation in the nonhospital setting. Transfusion. 1991; 31: 513-7.

16. Owings DV, Kruskall MS, Thurer RL, Donovan LM. Autologous blood donations prior to elective cardiac surgery. Safety and effect on subsequent blood use. Jama. 1989; 262: 1963-8.

17. Newman BH. Vasovagal reactions in high school students: findings relative to race, risk factor synergism, female sex, and non-high school participants. Transfusion. 2002; 42: 1557-60. 
18. Eder AF, Hillyer CD, Dy BA, Notari EPt, Benjamin RJ. Adverse reactions to allogeneic whole blood donation by 16- and 17-year-olds. JAMA. 2008; 299: 2279-86.

19. Laitinen T, Hartikainen J, Vanninen E, Niskanen L, Geelen G, Lansimies E. Age and gender dependency of baroreflex sensitivity in healthy subjects. J Appl Physiol. 1998; 84: 576-83.

20. Gribbin B, Pickering TG, Sleight P, Peto R. Effect of age and high blood pressure on baroreflex sensitivity in man. Circ Res. 1971; 29: 424-31.

21. Moya A, Sutton R, Ammirati F, Blanc JJ, Brignole M, Dahm JB, et al. Guidelines for the diagnosis and management of syncope (version 2009). Eur Heart J. 2009; 30: 2631-71.

22. Steptoe A, Sawada Y. Assessment of baroreceptor reflex function during mental stress and relaxation. Psychophysiology. 1989; 26: 140-7.

23. Ogata H, Iinuma N, Nagashima K, Akabane T. Vasovagal reactions in blood donors. Transfusion. 1980; 20: $679-83$

24. Takanashi M, Odajima T, Aota S, Sudoh M, Yamaga Y, Ono Y, et al. Risk factor analysis of vasovagal reaction from blood donation. Transfus Apher Sci. 2012; 47: 319-25.

25. Newman BH. Management of young blood donors. Transfus Med Hemother. 2014; 41: 284-95.

26. Eder AF, Dy BA, Kennedy JM, Notari Iv EP, Strupp A, Wissel ME, et al. The American Red Cross donor hemovigilance program: complications of blood donation reported in 2006. Transfusion. 2008; 48: 1809-19.

27. Wiltbank TB, Giordano GF, Kamel H, Tomasulo P, Custer B. Faint and prefaint reactions in whole-blood donors: an analysis of predonation measurements and their predictive value. Transfusion. 2008; 48: 1799-808. 


\section{Figure legend}

\section{Figure 1}

Distributions of mild, moderate, and severe vasovagal reactions (VVRs) by age in 4,192 autologous blood donations.

\section{Figure 2}

Incidence of VVRs by age. The VVR rate of 0-17-year-old patients was approximately three times as high as that of patients $18-89$ years old $(*: p<0.001)$. The VVR rate of $10-13$ year old patients was highest $(5 / 92$, $5.43 \%)$, followed by $14-17$-year-olds $(11 / 203,5.41 \%)$, and $18-19$ year-olds $(3 / 72,4.17 \%)$. In the adult population, susceptibility to VVRs was correlated negatively with increasing age with statistical significance (20-29 years: $5.0 \%, 30-39$ years: $4.0 \%, 40-49$ years: $1.6 \%, 50-59$ years: $1.4 \%, 60-69$ years: $0.8 \%$, and $70-79$ years: $0.5 \%, R=0.88, p=0.006)$. Error bars indicate $95 \%$ confidence intervals. 
Table 1. Patient Characteristics

\begin{tabular}{lll}
\hline Primary disease & $n$ (female/male) & Median age (range) (years) \\
\hline Total & $4,192(2,995 / 1,197)$ & $54(4-86)$ \\
Orthopedics & $2,050(1,618 / 432)$ & $57(4-86)$ \\
Gynecology/Obstetrics & $1,175(1,175 / 0)$ & $41(18-76)$ \\
Urology & $532(7 / 525)$ & $67(43-83)$ \\
Cardiovascular surgery & $247(87 / 160)$ & $53(8-83)$ \\
Dentistry & $81(48 / 33)$ & $52(16-52)$ \\
Other & $107(60 / 47)$ & $33.5(8-77)$ \\
\hline
\end{tabular}


Table 2. VVR predictors by univariate analysis

\begin{tabular}{|c|c|c|c|c|c|c|c|c|c|}
\hline \multirow{3}{*}{ Characteristic } & \multicolumn{2}{|c|}{ Group I } & \multirow{3}{*}{$p$ value } & \multirow{3}{*}{$\begin{array}{l}\text { non-VVR } \\
(n=2,500)\end{array}$} & \multicolumn{2}{|l|}{ Group II } & \multicolumn{2}{|c|}{ The overall group (Groups I + II) } & \multirow{3}{*}{$p$ value } \\
\hline & non-VVR & VVR & & & VVR & $p$ value & non-VVR & VVR & \\
\hline & $(n=1,605)$ & $(n=44)$ & & & $(n=43)$ & & $(n=4,105)$ & $(n=87)$ & \\
\hline $\begin{array}{l}\text { Age (years) } \\
\end{array}$ & $56(4-84)$ & $35(11-82)$ & $<0.0001$ & $53(5-86)$ & $36(10-74)$ & $\begin{array}{l}<0.0001 \\
\end{array}$ & $55(4-86)$ & $36(10-82)$ & $<0.0001$ \\
\hline BMI $\left(\mathrm{kg} / \mathrm{m}^{2}\right)$ & $22.3(13.5-44.0)$ & $20.4(15.1-26.1)$ & $<0.0001$ & $22.5(10.2-48.7)$ & $21.8(14.0-30.0)$ & 0.0928 & $22.4(10.2-48.7)$ & $21.3(14.0-30.0)$ & 0.0007 \\
\hline Systolic BP $(/ \mathrm{mmHg})$ & $135(91-198)$ & $120(101-147)$ & $<0.0001$ & $124(90-189)$ & $110(93-146)$ & $<0.0001$ & $128(90-198)$ & $117(93-147)$ & $<0.0001$ \\
\hline Weight $(\mathrm{kg})$ & $54.8(11.5-103.7)$ & $51.0(21.1-72.0)$ & 0.0002 & $56.0(15.0-110.0)$ & $55.0(17.4-78.0)$ & 0.0987 & $55.1(11.5-110.0)$ & $52.0(17.4-78.0)$ & 0.0016 \\
\hline $\operatorname{TBV}(\mathrm{L})$ & $3.38(1.19-5.73)$ & $3.22(1.89-4.82)$ & 0.008 & $3.85(0.62-7.45)$ & $3.76(0.78-5.38)$ & 0.1304 & $3.83(0.62-7.45)$ & $3.67(0.78-5.38)$ & 0.0115 \\
\hline Male/female & $468 / 1,137$ & $6 / 38$ & 0.025 & $715 / 1,785$ & $11 / 32$ & 0.664 & $1,183 / 2,922$ & $17 / 70$ & 0.058 \\
\hline First time collection & $867(54.0 \%)$ & $31(70.5 \%)$ & 0.031 & $1,472(58.9 \%)$ & $28(65.1 \%)$ & 0.410 & 2,339 (57.0\%) & $59(67.8 \%)$ & 0.043 \\
\hline Pulse (/bpm) & $74(45-127)$ & $79(53-103)$ & 0.039 & $74(45-140)$ & $81(52-111)$ & 0.9844 & $74(45-140)$ & $80(52-111)$ & 0.0019 \\
\hline Height (cm) & $157(100-185)$ & $158(118.3-173.5)$ & n.s. & & & & & & \\
\hline Diastolic BP (/mmHg) & $84(50-119)$ & $82(54-106)$ & n.s. & & & & & & \\
\hline WBC count $(/ \mu \mathrm{L})$ & $5,950(2,020-26,370)$ & $61,10(2,720-11,810)$ & n.s. & & & & & & \\
\hline $\mathrm{RBC}$ count $\left(10^{6} / \mu \mathrm{L}\right)$ & $420(214-816)$ & $423(349-552) \quad$ n.s. & & & & & & & \\
\hline Hemoglobin (g/dL) & $12.8(10.0-18.8)$ & 12.7 (10.7-17.2) n.s. & & & & & & & \\
\hline Hematocrit (\%) & $37.8(27.7-62.3)$ & $37.2(29.4-48.9)$ n.s. & & & & & & & \\
\hline Platelet count (//L) & $27.4(9.7-75)$ & 26.1 (15.7-50.1) n.s. & & & & & & & \\
\hline
\end{tabular}

BMI, body mass index; TBV, total blood volume; BP, blood pressure; WBC, white blood cell; RBC, red blood cell; n.s., not significant.

Data are presented as $n(\%)$ for male/female and first-time donation variables and as the median value (range) for all other variables. 
Table 3. VVR predictors by multivariate analysis

\begin{tabular}{|c|c|c|c|c|c|c|}
\hline \multirow[b]{2}{*}{ Variable } & \multicolumn{2}{|c|}{ Group I } & \multicolumn{2}{|c|}{ Group II } & \multicolumn{2}{|c|}{ The overall group (Groups I + II) } \\
\hline & OR $(95 \% \mathrm{CI})$ & $p$ value & OR $(95 \% \mathrm{CI})$ & $p$ value & OR $(95 \% \mathrm{CI})$ & $p$ value \\
\hline First-time collection & $2.76(1.31-5.81)$ & 0.007 & $1.44(0.76-2.72)$ & 0.265 & $1.81(1.12-2.92)$ & 0.015 \\
\hline Age $(<18$ years old $)$ & $2.77(1.16-6.61)$ & 0.021 & $1.00(0.36-2.74)$ & 0.993 & $1.76(0.92-3.34)$ & 0.086 \\
\hline Heart rate $(>74 / \mathrm{min})$ & $2.15(1.06-4.38)$ & 0.035 & $1.80(0.95-3.41)$ & 0.072 & $1.92(1.20-3.09)$ & 0.007 \\
\hline Systolic BP $(<120$ mmHg) & $2.17(1.05-4.49)$ & 0.037 & $4.23(2.04-8.76)$ & $<0.001$ & $2.45(1.50-4.01)$ & $<0.001$ \\
\hline Female gender & $2.22(0.72-6.86)$ & 0.166 & $0.77(0.36-1.67)$ & 0.508 & $1.27(0.68-2.36)$ & 0.452 \\
\hline Body mass index $\left(<22 \mathrm{~kg} / \mathrm{m}^{2}\right)$ & $1.64(0.74-3.66)$ & 0.224 & $1.21(0.61-2.43)$ & 0.574 & $1.51(0.90-2.53)$ & 0.118 \\
\hline Total blood volume $(<3.5 \mathrm{~L})$ & $0.70(0.28-1.75)$ & 0.448 & $0.67(0.32-1.40)$ & 0.291 & $0.72(0.41-1.25)$ & 0.242 \\
\hline
\end{tabular}

CI, confidence interval, OR, odds ratio. 


\section{(absolute number)}

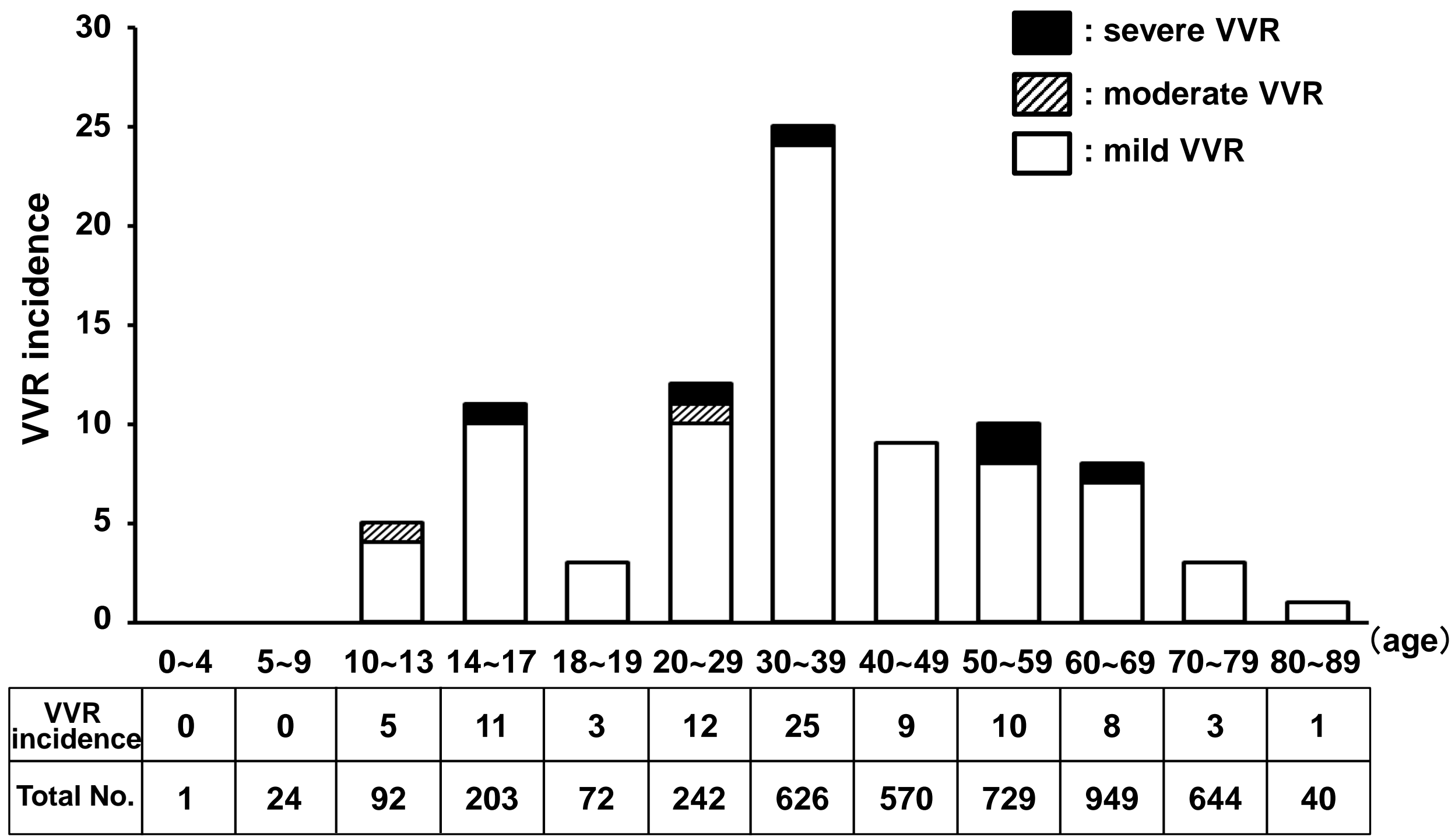

Figure 1 Nishimori et al. 


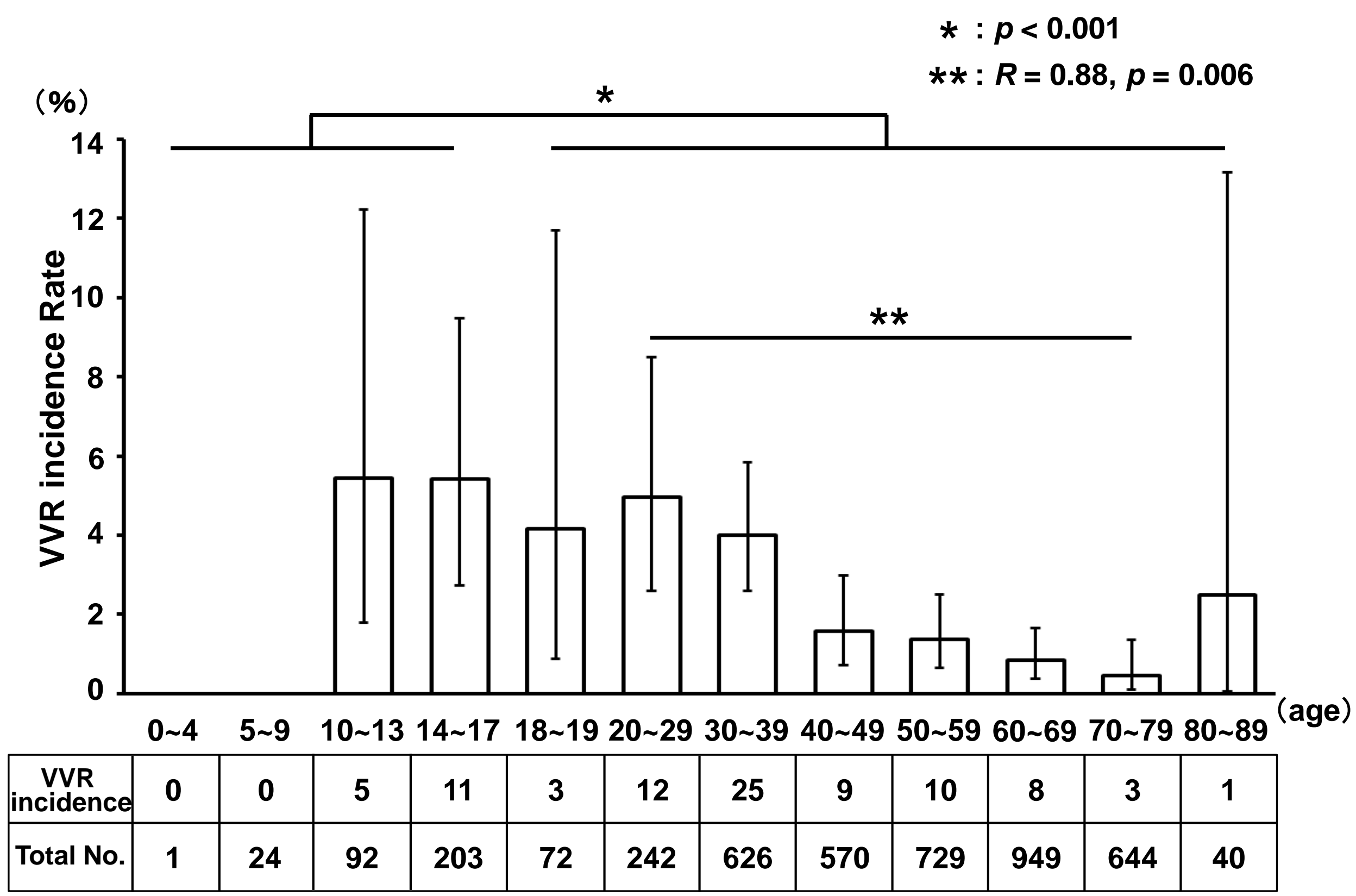

Figure 2 Nishimori et al. 